\title{
Surgical team turnover and operative time: An evaluation of operating room efficiency during pulmonary resection
}

\author{
Alain Joe Azzi, MD, ${ }^{a}$ Karan Shah, MSc, ${ }^{\mathrm{a}}$ Andrew Seely, MD, PhD, ${ }^{\mathrm{b}, \mathrm{c}}$ James Patrick Villeneuve, MD, PhD, \\ Sudhir R. Sundaresan, MD, ${ }^{\mathrm{b}}$ Farid M. Shamji, MD, ${ }^{\mathrm{b}}$ Donna E. Maziak, MD, MSc, ${ }^{\mathrm{b}, \mathrm{c}}$ and \\ Sebastien Gilbert, $\mathrm{MD}^{\mathrm{b}, \mathrm{c}}$
}

\begin{abstract}
Objective: Health care resources are costly and should be used judiciously and efficiently. Predicting the duration of surgical procedures is key to optimizing operating room resources. Our objective was to identify factors influencing operative time, particularly surgical team turnover.
\end{abstract}

Methods: We performed a single-institution, retrospective review of lobectomy operations. Univariate and multivariate analyses were performed to evaluate the impact of different factors on surgical time (skin-to-skin) and total procedure time. Staff turnover within the nursing component of the surgical team was defined as the number of instances any nurse had to leave the operating room over the total number of nurses involved in the operation.

Results: A total of 235 lobectomies were performed by 5 surgeons, most commonly for lung cancer $(95 \%)$. On multivariate analysis, percent forced expiratory volume in 1 second, surgical approach, and lesion size had a significant effect on surgical time. Nursing turnover was associated with a significant increase in surgical time (53.7 minutes; 95\% confidence interval, 6.4-101; $P=.026)$ and total procedure time (83.2 minutes; $95 \%$ confidence interval, 30.1-136.2; $P=.002$ ).

Conclusions: Active management of surgical team turnover may be an opportunity to improve operating room efficiency when the surgical team is engaged in a major pulmonary resection. (J Thorac Cardiovasc Surg 2016;151:1391-5)

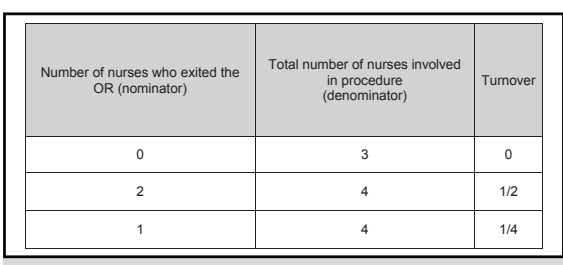

Examples of nursing turnover during a procedure.

Central Message

Active management of surgical team turnover may be an opportunity to improve operating room efficiency in major pulmonary resections.

\section{Perspective}

We report the impact of several factors on procedure times for major pulmonary resections, focusing on staff turnover within the surgical team. Our results highlight the importance of exploring human factors in surgical team dynamics in an effort to improve patient care and efficiency. We propose a novel definition of surgical team turnover that allows objective quantification as part of efforts to improve surgical quality.

See Editorial Commentary page 1396.
Lung cancer places a tremendous emotional and physical burden on patients and their families, and is a significant source of health care-related expenditures. ${ }^{1,2}$ Given that lung cancer resection is one of the most common procedures performed in general thoracic surgery, it is an appropriate target for attempts to improve efficiency in the use of operating room (OR) resources. ${ }^{3,4}$ Predicting the duration of lobectomies by relying solely on the surgeon's estimate has proven to be inadequate. ${ }^{5}$ Historical procedure time data also can be used to anticipate OR time

\footnotetext{
From the ${ }^{\mathrm{a}}$ Faculty of Medicine, University of Ottawa, ${ }^{\mathrm{b}}$ Division of Thoracic Surgery, University of Ottawa, and ${ }^{\mathrm{c}}$ The Ottawa Hospital Research Institute, Ottawa, Ontario, Canada.

Received for publication Oct 15, 2015; revisions received Dec 17, 2015; accepted for publication Dec 19, 2015; available ahead of print Feb 10, 2016.

Address for reprints: Sebastien Gilbert, MD, Division of Thoracic Surgery, University of Ottawa, The Ottawa Hospital, General Campus, Suite 6363, 501 Smyth Rd, Ottawa, Ontario K1H 8L6, Canada (E-mail: sgilbert@toh.on.ca). 0022-5223/ $\$ 36.00$

Copyright (c) 2016 by The American Association for Thoracic Surgery http://dx.doi.org/10.1016/j.jtcvs.2015.12.040
}

requirements. However, the accuracy of this predictor has been limited because it does not specifically address variability in the patient population and the OR work environment. $^{6}$ Our objective was to examine the relationships between operative times and patient-related factors, as well as surgical team factors, with the intention of optimizing health care resource use. Our main focus was turnover in OR team members during a major lung resection, not to be confused with OR turnover, which typically refers to the time between 2 separate procedures. Staff turnover is of particular interest as an opportunity to improve efficiency given the current paucity of evidence and lack of objective quantification. A high number of staff involved in a

Scanning this QR code will take you to the article title page. 


\section{Abbreviations and Acronyms \\ BMI = body mass index \\ CI = confidence interval \\ DLCO $\%=$ percent of diffusing capacity of the lungs for carbon monoxide \\ FEV $1 \%=$ percent of forced expiratory volume in 1 second \\ IQR = interquartile range \\ $\mathrm{OR} \quad=$ operating room \\ VATS $=$ video-assisted thoracoscopic surgery}

particular procedure has been used synonymously with high turnover. ${ }^{7,8}$ However, we propose a more precise definition and aim to examine potential links between surgical team dynamics and OR efficiency. Our hypothesis was that turnover within the surgical team would disrupt the flow of the operation and lead to decreased efficiency as reflected by longer operative times.

\section{MATERIALS AND METHODS}

After approval from the institutional Research Ethics Board, 254 patients who underwent lobectomy from January 2008 to December 2012 at a single institution were retrospectively reviewed. The following combined procedures were excluded: lobectomy combined with chest wall resection $(n=8)$, bronchoplasty $(n=2)$, arterioplasty $(n=4)$, and the use of a pleural tent $(n=5)$. The remaining 235 patients were analyzed. Patient-specific preoperative data, tumor characteristics, and procedural details were anonymized and stored in an encrypted database. Patient comorbidities were quantified using the Charlson Comorbidity Index. ${ }^{9}$ Total procedure time was defined as the period of time between entrance and exit of the patient from the OR. Surgical time was defined as the time from skin incision to skin closure. The surgical team consisted of nurses, residents, fellows, anesthesiologist, and surgeon involved in the operation. At our institution, nurses who are in the sterile field are registered practical nurses. We recognize that other health care professionals (eg, surgical technicians) may fulfill this role depending on institutional practices. The 1 to 2 anesthesia team member(s) typically involved in these operations were excluded from the count when analyzing the impact of team size on procedure times.

The definition of OR staff turnover was inspired from industry and modified to be relevant to the clinical setting. In assessing surgical team turnover, we evaluated nursing turnover, defined as the ratio of the number of nurses who exited the OR during the procedure to the total number of nurses involved in the procedure. Our definition of turnover results in a quantitative measurement. We provide examples of different scenarios and the resultant staff turnover in Table 1 . Nursing activity in and out of the OR for work-related breaks was recorded as turnover. Circulating nurses temporarily exiting the OR to obtain supplies were not considered turnover within the team. Procedures ending after the nursing day shift (ie, after 3:30 PM; 56/235 [24\%]) were specifically excluded from the turnover analysis because of the artificial increase in turnover activity resulting from this mandated shift change. The purpose of this exclusion criterion was to reduce the potential for bias in the relationship between turnover occurring during a regular workday and operative times.

Several other factors were examined using univariate and multivariate analysis for an effect on surgical time and total procedure time: age, body mass index (BMI), smoking history, Charlson comorbidity index, percent of forced expiratory volume in 1 second (FEV1\%), percent of diffusing capacity of the lungs for carbon monoxide (DLCO \%), tumor size, use of epidural catheter, surgical approach (video-assisted vs open), resected lobe, surgeon level of experience ( $\leq 10$ vs $>10$ years), surgical team size, presence of pleural adhesions, and the need for pulmonary decortication. Parameters were selected for inclusion in a multivariate linear regression model if the significance level of the univariate association with operative times was $\leq 0.2$. Pleural adhesions were used as a surrogate for previous surgery to the chest and previous chest irradiation. Operations were performed by a total of 5 surgeons. Surgeon experience was a potential predictor with a higher degree of surgeon specificity. We ensured that each surgeon contributed at least 40 consecutive operations. The intraclass correlation coefficients for operating surgeon were small for both surgical time $(0.9 \%)$ and total procedure time $(3.3 \%)$. Therefore, we did not include operating surgeon as a cluster in the analysis. ${ }^{10}$

\section{RESULTS}

Of the 235 patients included in the study, most were smokers aged 60 to 79 years (median, 67 years; interquartile range [IQR], 61-74) with a diagnosis of lung cancer ( $95 \%$; 223/235), a median BMI of 26 (IQR, 23-29), a median FEV1\% of $82 \%$ (IQR, 72-95), and a median DLCO of $75 \%$ (IQR, 65-86). The median tumor size was $2.7 \mathrm{~cm}$ (IQR, 2-4), and the right upper lobe was the most common location $(40 \% ; 94 / 235)$. Median comorbidity index was 5 (IQR, 4-6). An epidural catheter was used in $42 \%$ of cases (98/235). Pleural adhesions were reported in 40\% (94/235), and $15 \%(36 / 235)$ required partial pulmonary decortication in addition to lobectomy. A video-assisted thoracoscopic surgery (VATS) approach was used in 52\% (122/235) of cases. The median number of surgeons and residents was 3 (IQR, 2-3), and the median total number of nurses involved in a given operation was 3 (IQR, 3-4). There was no nursing staff turnover in $48 \%(113 / 235)$ of cases (median turnover ratio, 0 ; IQR, 0-0.33). The median surgical

TABLE 1. Examples of nursing turnover during a procedure

\begin{tabular}{lcc}
\hline Turnover scenarios & $\begin{array}{c}\text { Number of nurses who } \\
\text { exited the OR (nominator) }\end{array}$ & $\begin{array}{c}\text { Total number of nurses } \\
\text { involved in procedure } \\
\text { (denominator) }\end{array}$ \\
\hline $\begin{array}{l}\text { Three nurses are involved in the case. No nurse leaves or enters the OR } \\
\text { Three nurses are involved in the case. No nurse leaves. One additional } \\
\text { nurse enters the OR halfway through the case }\end{array}$ & 0 & 3 \\
$\begin{array}{l}\text { Three nurses are involved in the case. One nurse leaves halfway through } \\
\text { the case and is replaced by another nurse }\end{array}$ & 0 & 4 \\
\hline OR, Operating room. & & 0 \\
\end{tabular}

$O R$, Operating room. 
time was 184 minutes (IQR, 178-202), and the median total procedural time was 241 minutes (IQR, 235-261).

The results of the univariate and multivariate analyses are summarized in Table 2. On univariate analysis, factors associated with a significant increase in surgical time were BMI (1.4 minutes per unit increase; $95 \%$ confidence interval $[\mathrm{CI}], 0.1-2.7 ; P=.035)$, FEV1\% (0.5 minutes per $1 \%$ decrease; 95\% CI, 0.1-0.8; $P=.013$ ), tumor or lesion size $(3.6$ minutes per $1 \mathrm{~cm} ; 95 \% \mathrm{CI}, 0.2-6.9 ; P=.036)$, and the presence of pleural adhesions (22.4 minutes; $95 \%$ CI, 9.6-35.1; $P=.001)$.

Factors associated with a significantly prolonged total procedure time were BMI (1.9 minutes; $95 \%$ CI, 0.5-3.2; $P=.008)$, FEV1\% (0.5 minutes per $1 \%$ decrease; $95 \%$ CI, 0.08-0.8; $P=.019)$, lesion size (5.2 minutes per $1 \mathrm{~cm}$ increase; 95\% CI, 1.7-8.8; $P=.004$ ), pleural adhesions (22.5 minutes; 95\% CI, 8.7-36.3; $P=.002$ ), need for pulmonary decortication (18.3 minutes; $95 \%$ CI, 0.4-36.2; $P=.045$ ), and nursing staff turnover (57.8 minutes; $95 \%$ CI, $8-107 ; P=.023$ ). Patient comorbidity, age, smoking history, DLCO $\%$, use of an epidural catheter, surgical team size, resected pulmonary lobe, and surgeon experience had no significant impact on surgical or total procedure time. The VATS approach was associated with longer surgical times, but the univariate relationship did not reach statistical significance (11.9 minutes; $95 \%$ CI, -0.03 to 23.9 ; $P=.051)$.

On multivariate analysis, FEV1\%, VATS approach, and lesion size were significantly associated with prolonged surgical time (Table 2). The presence of pleural adhesions led to a significant increase in both surgical time and total procedure time. Finally, turnover within the nursing component of the surgical team remained associated with a significant increase in total procedure time ( 83.2 minutes; $95 \%$ CI, 30.1-136.2; $P=.002)$ and significantly prolonged surgical time (53.7 minutes; 95\% CI, 6.4-101; $P=.026$ ).

\section{DISCUSSION}

The observed correlations among BMI, FEV1\%, tumor size, and operative times in the univariate analysis can be rationalized easily by anyone on the surgical team who has experienced intraoperative challenges related to obesity, chronic obstructive pulmonary disease, and resection of larger tumors. Previous studies have demonstrated a relationship between operative efficiency and age, gender, American Society of Anesthesiologists score, and surgical team experience. ${ }^{11-14}$ These findings could not be replicated in our study population, composed entirely of patients undergoing lung resection. However, our results corroborate the findings of a systematic review of general thoracic surgery procedures reporting that age, gender, tobacco use, and comorbidities (including American Society of Anesthesiologists score) had minimal or no significant impact on surgical times. ${ }^{15}$ More recent evidence indicates that increasing BMI and surgical team size may independently lead to prolonged operating times. ${ }^{7,8,16}$ We have found that the effect of BMI and team size on operative time was no longer significant when examined in a multivariate analysis. We think it is worthwhile to gather information on the relationship between nonmodifiable, patient-related factors and procedure times for several reasons: (1) to identify potential

TABLE 2. Factors influencing operative times $(\mathbf{n}=\mathbf{2 3 5})$

\begin{tabular}{|c|c|c|c|c|}
\hline & $\begin{array}{c}\text { Mean increase } \\
\text { Surgical time, } \min (95 \% \mathrm{CI})\end{array}$ & $\boldsymbol{P}$ & $\begin{array}{c}\text { Mean increase } \\
\text { Total procedure time, } \min (95 \% \mathrm{CI})\end{array}$ & $P$ \\
\hline \multicolumn{5}{|l|}{ Univariate } \\
\hline BMI & $1.4(0.1-2.7)$ & .035 & $1.9(0.5-3.2)$ & .008 \\
\hline FEV1 $\%$ (per $1 \%$ decrease) & $0.5(0.1-0.8)$ & .013 & $0.5(0.08-0.8)$ & .019 \\
\hline VATS vs thoracotomy & $11.9(-0.03$ to 23.9$)$ & .051 & $-3.8(-16.8$ to 9.2$)$ & .566 \\
\hline Lesion size (per $1 \mathrm{~cm}$ ) & $3.6(0.2-6.9)$ & .036 & $5.2(1.7-8.8)$ & .004 \\
\hline Surgical team size (per person) & $4.1(-4.9$ to 13.0$)$ & .370 & $7.8(-2.0$ to 17.6$)$ & .119 \\
\hline Nursing staff turnover & $42.0(-3.5$ to 87.5$)$ & .070 & $57.8(8.0-107.6)$ & .023 \\
\hline Need for decortication & $12.6(-4.1$ to 29.3$)$ & .137 & $18.3(0.4-36.2)$ & .045 \\
\hline Pleural adhesions & $22.4(9.6-35.1)$ & .001 & $22.5(8.7-36.3)$ & .002 \\
\hline \multicolumn{5}{|l|}{ Multivariate* } \\
\hline BMI & $1.3(-0.3$ to 2.8$)$ & .109 & $1.4(-0.4$ to 3.1$)$ & .112 \\
\hline FEV $1 \%$ (per $1 \%$ decrease) & $0.5(0.01-1.0)$ & .044 & $0.5(-0.006$ to 1.1$)$ & .052 \\
\hline VATS vs thoracotomy & $18.1(3.2-32.9)$ & .018 & - & - \\
\hline Lesion size (per 1 unit) & $4.9(0.5-9.3)$ & .029 & $5.1(0.2-9.9)$ & .040 \\
\hline Surgical team size (per person) & - & - & $10.9(-0.7$ to 22.5$)$ & .065 \\
\hline Nursing staff turnover & $53.7(6.4-101.0)$ & .026 & $83.2(30.1-136.2)$ & .002 \\
\hline Need for decortication & $-10.5(-35.4$ to 14.3$)$ & .403 & $-3.9(-31.3$ to 23.6$)$ & .781 \\
\hline Pleural adhesions & $18.92(1.6-36.3)$ & .033 & $14.9(-4.7$ to 33.5$)$ & .139 \\
\hline
\end{tabular}

Numbers in bold are statistically significant $P$ values $(P<.05)$. CI, Confidence interval; BMI, body mass index; $F E V 1 \%$, percent of forced expiratory volume in 1 second; VATS, video-assisted thoracoscopic surgery. *The multivariate analysis only included preoperative factors with a $P<.2$ on univariate analysis. 
confounding factors for the relationship between turnover and operative time; (2) to externally verify the potential impact of these factors at our institution; and (3) to develop a data-driven approach to our case scheduling protocols (eg, avoid scheduling patients with multiple factors prolonging operative time on the same day). As outlined earlier, conflicting evidence as to which factors significantly affect operative times underscores the need for more controlled studies.

To our knowledge, turnover within the surgical team has not been quantified clearly or measured in recent literature on OR efficiency. By objectively quantifying turnover, we were able to measure its impact on operative times, which we used as a surrogate measure of efficiency. When we analyzed the data, we found no instances of surgeon, surgical fellow, or resident turnover. Likewise, opportunities for turnover of anesthesia team members are few. Anesthesia care delivery within our health system may be distinct from others in that the same anesthesiologist is not responsible for more than 1 ongoing major operation. During a typical procedure, the anesthesiologist is usually working in conjunction with 1 resident or as the sole anesthesia care provider. Therefore, we focused the turnover analysis on nurse team members. We would like to emphasize the importance of nurses as core members of the surgical team and their overwhelmingly positive impact on OR efficiency. As part of our collective goal to improve surgical quality, all members of the team are committed to an ongoing quest for potential ways to improve efficiency. We analyze our behavior and workflow with a focus on data-driven, constructive, team-building activities where every team member has a voice. This approach is aligned with the objectives set out by the Comprehensive Unitbased Safety Program of the American College of Surgeons.

The observed relationship between turnover and operative time raises questions regarding potential mechanisms of action. When surgical staff leave the OR or are replaced, there is potential for communication breakdown that may affect overall team performance. For instance, if the surgeon requests that the team prepare for an upcoming portion of the operation and there is a communication gap as the circulating nurse is temporarily relieved from duty, the progression of the surgery may be interrupted. This phenomenon could also affect the concentration and focus of the team and lead to further delays. Another potential explanation of the effect of turnover could be that team members who are present throughout a major procedure can better anticipate the next steps that will be required to maintain the flow of the operation. On that basis, efficiency could be hindered when members of the team leave the operation while critical portions are ongoing. The simplest way to imagine the potential effects of staff turnover on efficiency is to use the extreme example of the end of a nursing day shift. At that time, there is usually near complete staff turnover and instruments must be counted again. Our institutional policy, which is based on recommendations from national nursing associations, is to have the scrub nurse who is being relieved continue to work in the sterile field while the recount is being performed by the new scrub nurse and the circulating nurse. We understand that the instrument recount is implemented as a safety measure. However, it can create unrealistic demands on team members if they are asked to perform more than 1 task at the same time. For instance, the scrub nurse being relieved may have to assist the new scrub nurse with the instrument count and, at the same time, be expected to focus on the ongoing procedure. In a similar manner, the circulating nurse may not be available to address a request from the surgeon while trying to accurately perform the instrument recount. We eliminated procedures where a shift change occurred from the analysis to reduce the potential for bias and to provide a more objective description of the relationship between turnover and procedure time as it unfolds during most cases. As expected, the mean turnover ratio was significantly higher in this subgroup of procedures. Inclusion of cases where there was a planned shift change would have artificially increased turnover. Excluding these procedures also was helpful in preserving the simplicity of the turnover definition by avoiding exceptions based on procedure end time and in improving the generalizability of the results to institutions that may not experience these specific constraints.

We think it is possible to explore ways of improving efficiency while maintaining the highest level of patient safety, respecting employment regulations, and providing a healthy work environment for all. Improving timeliness in completing surgical procedures can have positive economic repercussions because it can reduce overtime pay and decrease cost per weighted case by increasing the number of procedures performed in the available OR time. There are potential strategies to minimize turnover during a surgical procedure. According to our proposed definition, the addition of staggered work shifts with a focus on relieving nurses already engaged in surgery would effectively reduce the turnover ratio while preserving the level of human resources within the surgical team. Another potential approach would be to purposefully delay 1 or more mandatory work breaks until after the procedure is completed. We have had some success in implementing this measure with our scrub nurses often volunteering to work continuously until the procedure has been completed. A relatively minor extension of our regular OR day shift schedule could be a potential solution to address the turnover associated with shift change. One of our previous pilot projects showed that more than half of the operative room days finishing late would have been completed on time, without the need for staff turnover, if an extra 30 minutes had been added to the day shift. Some of the proposed 
solutions imply large-scale changes to work routines and culture. Therefore, we would like to underscore the importance of engaging all potential stakeholders, including hospital administrators who should be invited to join the efficiency project team from its inception.

\section{Study Limitations}

We acknowledge that the results are subject to the forms of bias associated with retrospective reviews. There may be other potentially important factors for which information was not available for review. For instance, our institution is engaged in the training of OR nurses. New OR nurses undergoing orientation will be exposed to thoracic surgery and are typically added as an extra member of the team to work with more experienced nurses. The record does not indicate the level of experience of nurses, and we could not measure the impact of the addition of a nurse in orientation, if any, on turnover. We also recognize that it is difficult to extrapolate results from a single-center experience. Proof of generalizability of our quantitative approach to staff turnover will require external validation by other investigators. Given the complex set of interactions occurring during a major thoracic surgery procedure, other potential confounding factors, such as prior thoracic irradiation or extent of mediastinal lymphadenectomy, were not specifically accounted for. However, we documented the effect of pleural adhesions, which may act as a surrogate for a history of thoracic radiation. We reserve complete mediastinal lymph node dissection, which can prolong procedure time, for patients with $\mathrm{N} 2$ disease who are deemed candidates for surgery. The latter constitute a subset of the study population that is too small to perform a significant stratified analysis. We are also aware that we could not account for other types of delays related to patient factors and to the surgery or anesthesia components of the procedure (eg, difficult arterial line access, difficult airway for intubation, poor lung deflation despite adequate lung isolation, spontaneous breathing or coughing during surgery, suboptimal efficiency in surgical technique, intraoperative dislodgement of the double-lumen endotracheal tube). Without reliable data and defined quantitative methodology, we cannot determine if these factors led to any significant bias in the statistical analysis.

\section{CONCLUSIONS}

The performance self-evaluation of a surgical team should take into account a multiplicity of factors, including procedure times as a measure of efficiency. It is understood that all members of the surgical team are dedicated to the welfare of the patient and that lapses in efficiency may not always be within their control. Collaboration between health care providers and managers should focus on promoting open communication while attempting to identify challenges and find solutions to optimize workflow. Active management of turnover within the surgical team probably should be part of this process. The results highlight the importance of exploring the impact of human factors on surgical team dynamics as part of quality-improvement strategies aimed at more efficient use of costly and often limited health care resources.

\section{Conflict of Interest Statement}

Authors have nothing to disclose with regard to commercial support.

We would like to recognize the dedication and patience of all our OR nurses, with special thanks to Angie Stackhouse, Kristin Waddell, Natalie Tomas, Karen Moore, Genevieve Malarczyk, and Lisa Young. Many thanks also to our research team, Kaitlyn Chambers, Caitlin Anstee, Elham Sabri, and Anna Fazekas, for their assiduity and unwavering support.

\section{References}

1. Kutikova L, Bowman L, Chang S, Long SR, Obasaju C, Crown WH. Economic burden of lung cancer and the associated costs of treatment failure in US. Lung Cancer. 2005;50:143-54.

2. Montazeri A. Quality of life data as prognostic indicators of survival in cancer patients: an overview of the literature from 1982 to 2008. Health Qual Life Outcomes. 2009;7:102.

3. Little AG, Rusch VW, Bonner JA, Gaspar LE, Green MR, Webb WR, et al. Patterns of surgical care of lung cancer patients. Ann Thorac Surg. 2005;80:2051-6.

4. Bendzsak A, Nenshi R, Darling G, Schultz SE, Gunraj N, Wilton AS, et al. Overview of lung cancer surgery in Ontario. Ann Thorac Surg. 2010;91:361-6.

5. Laskin DM, Abubaker O, Strauss RA. Accuracy of predicting the duration of a surgical operation. J Oral Maxillofac Surg. 2013;71:446-7.

6. Pandit JJ, Carey A. Estimating the duration of common elective operations: implications for operating list management. Anaesthesia. 2006;61:768-76.

7. Cassera MA, Zheng B, Martinec DV, Dunst CM, Swanstrom LL. Surgical time independently affected by surgical team size. Am J Surg. 2009;198:216-22.

8. Zheng B, Panton ON, Al-Tayeb TA. Operative length independently affected by surgical team size: data from 2 Canadian hospitals. Can J Surg. 2012;55:371-6.

9. Charlson ME, Pompei P, Ales KL, Mackenzie CR. A new method of classifying prognostic comorbidity in longitudinal studies: development and validation. $J$ Chron Dis. 1987;40:373-83.

10. Bell BA, Ene M, Smiley W, Schoeneberger JA. A multilevel primer using SAS ${ }^{\circledR}$ PROC MIXED. Presented at: SAS Glob Forum 2013 Proceedings; April 28-May 1, 2013; San Francisco, CA.

11. Strum DP, Sampson AR, May JH, Vargas LG. Surgeon and type of anesthesia predict variability in surgical procedure times. Anesthesiology. 2000;92:1454-66.

12. Papandria D, Rhee D, Ortega G, Zhang Y, Gorgy A, Makary MA, et al. Assessing trainee impact on operative time for common general surgical procedures in ACS-NSQIP. J Surg Educ. 2012;69:149-55.

13. Burt BM, ElBardissi AW, Huckman RS, Cohn LH, Cevasco MW, Rawn JD, et al Influence of experience and the surgical learning curve on long term patient outcomes in cardiac surgery. J Thorac Cardiovasc Surg. 2015;150:1061-8.

14. Enter DH, Lou X, Hui DS, Andrei AC, Barner HB, Sheen L, et al. Practice improves performance on a coronary anastomosis stimulator, attending surgeon supervision does not. J Thorac Cardiovasc Surg. 2015;149:12-6.

15. Dexter F, Dexter EU, Masursky D, Nussmeier NA. Systematic review of genera thoracic surgery articles to identify predictors of operating room case durations. Anesth Analg. 2008;106:1232-41.

16. St Julien JB, Aldrich MC, Sheng S, Deppen SA, Burfeind WR Jr, Putnam JB, et al. Obesity increases operating room time for lobectomy in the Society of Thoracic Surgeons database. Ann Thorac Surg. 2012;94:1841-7.

Key Words: surgical quality, efficiency, management, staff turnover, lung surgery, health economics 